\title{
ESQUEMA CORPORAL: UNA CONDUCTA BÁSICA PARA EL APRENDIZAJE DEL NIÑO CON AUTISMO
}

\author{
Hannia Cabezas Pizarro \\ Docente de la Universidad de Costa Rica \\ hcabezas@cariari.ucr.ac.cr
}

Recibido 30-VIII-2004 • Aceptado 7-IX-2004

\begin{abstract}
Resumen: En esta investigación se trabaja el esquema corporal en cinco niños con autismo. Dentro de los principales repertorios conductuales se incorpora el reconocimiento del "yo", de la nariz, boca, ojos, y cabeza. Se llevan a cabo sesiones sistemáticas, en las que se aplica la terapia conductual como un medio para alcanzar los porcentajes de respuesta deseados. Los datos obtenidos durante las sesiones se representan a través de cuadros y gráficos, lo que permite visualizar los avances de cada niño. Se utiliza el diseño de sujeto único y criterio cambiante, en donde "el experimentador cambia sucesivamente el criterio para la obtención de la consecuencia, usualmente en pasos graduados desde la línea base hasta el nivel terminal deseado." (Hall, 1973) p. 23.
\end{abstract}

Palabras clave: Educación, esquema corporal, terapia cognitivo conductual, autismo.

\begin{abstract}
This article discusses a study of the body system of five autistic children. It incorporates the recognition of body parts nose, eyes, and head as well as behavioral repertoires such as the "ego". The Behavioral Therapy was applied, in systematic sessions, as a way to reach the percentage of expected response. The data obtained during these sessions is presented in graphs. In order to see the process and the advance in each of the children, the researcher used the "unique subject and changing criteria methodology" in which the investigator changes the criteria successively to reach the consequence wanted. This is usually done in gradual steps from the base line to the desired final level (Hall,1973: 23).
\end{abstract}

Key words: Education, body system, behavioral therapy, autism.

\section{Introducción}

La elaboración del aprendizaje, se sustenta en el conocimiento de nuestro cuerpo, toda conducta la incorporamos a través de él. Los niños con autismo han mostrado una deficiencia en esta área, siendo una de sus dificultades la poca capacidad para el reconocimiento de su yo, y la inversión pronominal características apuntadas por Kanner (1943) y otros investigadores.

El adquirir estas habilidades es indispensable para interactuar con el mundo que nos rodea, y es por ello que este estudio se dirige hacia la enseñanza de la identificación del "yo" y otras partes del cuerpo como: la nariz, la boca ojos y cabeza de cinco niños con autismo, conductas que son indispensables para la incorporación del resto del aprendizaje.

El concepto de esquema corporal no es nuevo, Marleau (1945) se refiere a él como la forma de expresar que el cuerpo ocupa un lugar en el espacio, y que este espacio del cuerpo propio, es situacional, ya que existe, hacia las tareas que desarrolla y en las que participa.

El concepto que tengamos de él es el eje sobre el que se sustenta el aprendizaje. 
Aquí entran en juego tanto los factores físicos como los niveles de percepción visual y auditiva de cada persona detectándose que "hay una coexistencia de las perturbaciones: en el esquema corporal, en las relaciones espacio-temporales, en las alteraciones psicomotoras y en las deficiencias en el lenguaje. Todas estas llevan a las dificultades en el proceso de aprendizaje." (Hernández, 2004, p. 15) de allí la importancia que toma el descubrimiento del cuerpo por parte de los niños, asociado al aprendizaje.

En este estudio la primera conducta que se trabajó fue el "concepto de yo", para que los niños se identificaran como personas, y a partir de este concepto se les llevó a la identificación de otras partes como: la cabeza, la boca, la nariz y los ojos.

Se describen los procedimientos conductuales utilizados y se apunta el seguimiento sesión por sesión hasta alcanzar los niveles deseados.

\section{Trastorno autista y esquema corporal}

La definición de autismo ha ido evolucionado a través del tiempo, desde Kanner (1943) que fue el primero en dar una caracterización precisa la que ha sido enriquecida por las diferentes investigaciones que han permitido describir un cuadro clínico más completo y comprensivo del mismo (Tinbegem, 1962; Lovaas, 1965; Polaino, 1981; Hobson, 1995) en donde, este síndrome puede manifestarse de diferentes formas, y variar en grado e intensidad

El Manual diagnóstico y estadístico de los trastornos mentales (1995) lo define como:

\footnotetext{
"un trastorno generalizado del desarrollo que se caracteriza por una perturbación grave y generalizada de varias áreas del desarrollo: habilidades para la interacción social, habilidades para la comunicación o la presencia de comportamientos, intereses y actividades estereotipadas. Las características esenciales del trastorno autista son la presencia de un desarrollo marcadamente anormal o deficiente de la
}

interacción y comunicación sociales y un repertorio sumamente restringido de actividades e intereses" (1995, p. 69).

Al ser descrito el autismo como un problema profundo, el proceso normal de desarrollo, no se da en el niño de la misma forma que en el resto de la población. Las conductas no son adquiridas paulatina y en forma conciente como un acto madurativo ni los conceptos se construyen por la capacidad propia del niño, debido a que el pensamiento no es regulado por la representación mental sino a través de estimulaciones externas que pueden inducirse por programas conductuales comprensivos.

El desarrollo del lenguaje depende de procesos cognitivos, descubrimiento llevado a cabo desde hace tiempo por diferentes autores. (Vygotski, 1979; Luria, 1978).

Así mismo el descubrimiento del cuerpo y su funcionalidad va a estar ligado al conocimiento del lenguaje y la capacidad para comprender su significado.

Al adquirirse el lenguaje se da una reorganización mental, porque a través de la palabra se forman las representaciones mentales y se desarrollan otras áreas importantes como son la atención y la memoria. (Vygotski, 1979).

El lenguaje se da en forma paulatina y ordenada. Conforme el niño madura, en los primeros meses "la actividad es meramente instrumental. Más tarde se interioriza y, mediante su relación con los demás se incorpora a la cultura, convirtiéndose en mediación significativa" (Gallardo y Gallego, 1993).

Para esta acción el niño debe estar en contacto con el medio inmediato, la cercanía con la madre juega un papel importante para desarrollar conductas de apego y afectivas, convirtiéndose el contacto ocular en un canal fundamental para incorporar esta habilidad. Al carecer el niño con autismo de esta destreza la tarea se dificulta más, ya que no responde en forma adecuada a los diferentes sonidos del medio, ni busca a la persona que le habla como una reacción a la voz humana. Es a través de 
estas manifestaciones que en el proceso de desarrollo del lenguaje el niño inicia una comunicación con el adulto.

$\mathrm{Al}$ descubrir el niño el mundo que le rodea, establece las relaciones entre él y las personas y a través del reconocimiento de su cuerpo construye su espacio y sus límites, tomando conciencia de cada una de sus partes con ayuda de los sentidos.

Cobra vital importancia el descubrimiento del esquema corporal que ha sido definido como: "la toma de conciencia global del cuerpo que permite simultáneamente el uso de determinadas partes de él, así como conservar su unidad en las múltiples acciones que puede ejecutar" (Condemarín, 1995, p. 182).

En la medida en que el niño reconoce su propio cuerpo y lo domina, se facilita la incorporación de los aprendizajes, pasando a ser el punto de partida para la orientación en el espacio.

"La evolución de la actividad motriz se hace en el espacio en relación con el cuerpo. El cuerpo, en sí mismo, está orientado en un espacio de actividad antes de tener conocimiento de los componentes de su cuerpo y de verbalizar sus nombres correspondientes." (Condemarín, 1995, p. 183).

Fernández (1994) elabora dos conceptos más asociados al esquema corporal, que el niño desarrolla, y a los que denomina el espacio de la realidad y el espacio de la intención del deseo. Es así como la percepción de sí mismo, y el reconocimiento del cuerpo se convierten en herramientas indispensables para la adquisición de otros conceptos, como: relaciones de desplazamiento corporales, identificación del espacio y asociación de objetos básicos para el aprendizaje.

\section{Metodología}

Se trabajó con cinco niños con diagnóstico de autismo. El programa se basó en la teoría del reforzamiento (Skinner, 1943; Ribes Iñesta, 1984). Se utilizaron reforzadores primarios y sociales contingentes a la emisión de la conducta esperada.

Se llevaron a cabo registros sistemáticos para establecer una medida cuantificable sobre las conductas trabajadas. Se determinó la línea base para cada conducta que se iba a intervenir, la que se ha definido como "el registro del nivel operante de la conducta" (Hall, 1973, p. 11).

Se utilizó el diseño de criterio cambiante en donde

"el experimentador cambia sucesivamente el criterio para la obtención de la consecuencia, usualmente en pasos graduados, desde la línea base hasta el nivel terminal deseado. Si la conducta cambia sucesivamente de acuerdo a los niveles fijados o se aproxima, puede demostrarse control experimental.” (Hall, 1973, p. 23).

\section{Resultados}

El análisis de los resultados, se presenta a través de tablas y gráficos, lo que permite visualizar en forma total el proceso. Los resultados obtenidos en las primeras dos sesiones representados en cada una de las tablas, corresponden a la línea base y a partir de la tercera sesión se introducen los procedimientos de intervención, en donde se puede observar un incremento sistemático de la conducta que se está trabajando.

El promedio de sesiones para la adquisición de cada una de las conductas fue de 11 por sujeto por conducta, para un total de 227 sesiones cuyo tiempo de duración fue una hora.

La identificación de sí mismo, y la toma de conciencia del cuerpo son elementos importantes que le permiten al niño una mejor adaptación al mundo que les rodea. Como puede observarse en los datos registrados en la tabla $\mathrm{N}^{\circ} 1$ el promedio de la línea base para los cinco niños fue de $11,5 \%$, lo que nos indica que ninguno de ellos tenía el concepto de yo.

Los niños $\mathrm{N}^{\circ} 3$ y $\mathrm{N}^{\circ} 4$ lo incorporaron a partir de las sesiones quinta y sexta, el $\mathrm{N}^{\circ} 2$ de la doceava el $\mathrm{N}^{\circ} 1$ de la catorceava y el $\mathrm{N}^{\circ} 5$ no adquirió esta conducta. La 
Tabla $\mathrm{N}^{\circ} 1$

Concepto de "Yo"

Porcentajes de respuesta

\begin{tabular}{lccccc}
\hline $\begin{array}{l}\text { No de } \\
\text { sesión }\end{array}$ & $\begin{array}{c}\text { Sujeto } \\
1\end{array}$ & $\begin{array}{c}\text { Sujeto } \\
2\end{array}$ & $\begin{array}{c}\text { Sujeto } \\
3\end{array}$ & $\begin{array}{c}\text { Sujeto } \\
4\end{array}$ & $\begin{array}{c}\text { Sujeto } \\
5\end{array}$ \\
\hline L.base 1 & 0 & 0 & 35 & 20 & 0 \\
2 & 0 & 0 & 40 & 20 & 0 \\
intervención & & & & & \\
3 & 0 & 10 & 50 & 63 & 0 \\
4 & 0 & 44 & 40 & 50 & 0 \\
5 & 5 & 93 & 65 & 70 & 0 \\
6 & 0 & 40 & 100 & 100 & 0 \\
7 & 13 & 65 & 100 & 100 & 5 \\
8 & 7 & 29 & 100 & 100 & 0 \\
9 & 30 & 60 & 100 & 100 & 10 \\
10 & 56 & 30 & & & 0 \\
11 & 53 & 49 & & & 20 \\
12 & 60 & 90 & & & 20 \\
13 & 50 & 80 & & & 20 \\
14 & 75 & 90 & & & 10 \\
15 & 80 & 95 & & & 30 \\
16 & 75 & 95 & & & 40 \\
\hline
\end{tabular}

variabilidad de los resultados están correlacionados con la severidad del síndrome de cada uno de los niños

Es importante apuntar que al ser el cuerpo el punto de partida para los aprendizajes, y el reconocimiento e identidad del mismo un factor esencial para la incorporación del conocimiento, la enseñanza de los conceptos, la relación de espacio, concepto de sí mismo y funcionamiento general, es por lo que esta conducta se torna vital en el proceso educativo de los niños con autismo.

Tabla $\mathrm{N}^{\circ} 2$

Tóquese la nariz

Porcentajes de respuesta por sesión

\begin{tabular}{|c|c|c|c|c|c|c|}
\hline \multicolumn{2}{|l|}{$\begin{array}{l}\text { No de } \\
\text { sesión }\end{array}$} & $\begin{array}{c}\text { Sujeto } \\
1\end{array}$ & $\begin{array}{c}\text { Sujeto } \\
2\end{array}$ & $\begin{array}{c}\text { Sujeto } \\
3\end{array}$ & $\begin{array}{c}\text { Sujeto } \\
4\end{array}$ & $\begin{array}{c}\text { Sujeto } \\
5\end{array}$ \\
\hline $\begin{array}{l}\text { Línea } \\
\text { base }\end{array}$ & $\begin{array}{l}1 \\
2\end{array}$ & $\begin{array}{l}0 \\
0\end{array}$ & $\begin{array}{l}0 \\
0\end{array}$ & $\begin{array}{l}30 \\
20\end{array}$ & $\begin{array}{l}20 \\
40\end{array}$ & $\begin{array}{l}0 \\
0\end{array}$ \\
\hline \multicolumn{7}{|l|}{ inter- } \\
\hline & 4 & 40 & 15 & 80 & 47 & 5 \\
\hline & 5 & 47 & 25 & 70 & 70 & 0 \\
\hline & 6 & 70 & 10 & 90 & 50 & 5 \\
\hline & 7 & 80 & 21 & 100 & 50 & 0 \\
\hline & 8 & 75 & 40 & 100 & 70 & 10 \\
\hline & 9 & 85 & 90 & 100 & 80 & 15 \\
\hline & 10 & 90 & 100 & 100 & 80 & 20 \\
\hline & 11 & 90 & 100 & & 90 & 10 \\
\hline & 12 & 95 & 90 & & 100 & \\
\hline & 13 & & 90 & & 100 & \\
\hline & 14 & & 90 & & 100 & \\
\hline & 15 & & & & 100 & \\
\hline
\end{tabular}

Al identificarse a sí mismos, y utilizar la primera persona para referirse a ellos, les permitió una mejor comunicación con el terapeuta, desarrollaron habilidades motoras más finas como el señalar e identificar con su dedo índice la nariz. Este proceso abrió paso a que asociaran la funcionalidad de la nariz y discriminaran diferentes olores.

Gráfico $\mathrm{N}^{\circ} 1$

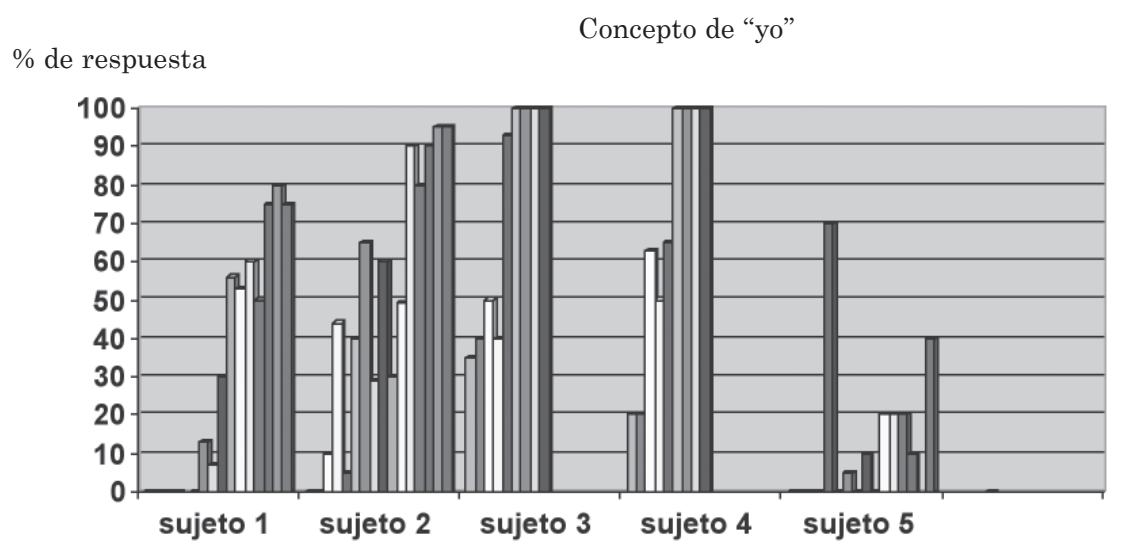

No. de sesión

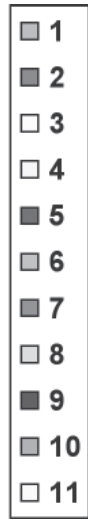




\section{Gráfico $N^{\circ} 2$}

Orden: "Tóquese la nariz."

$\%$ de respuesta

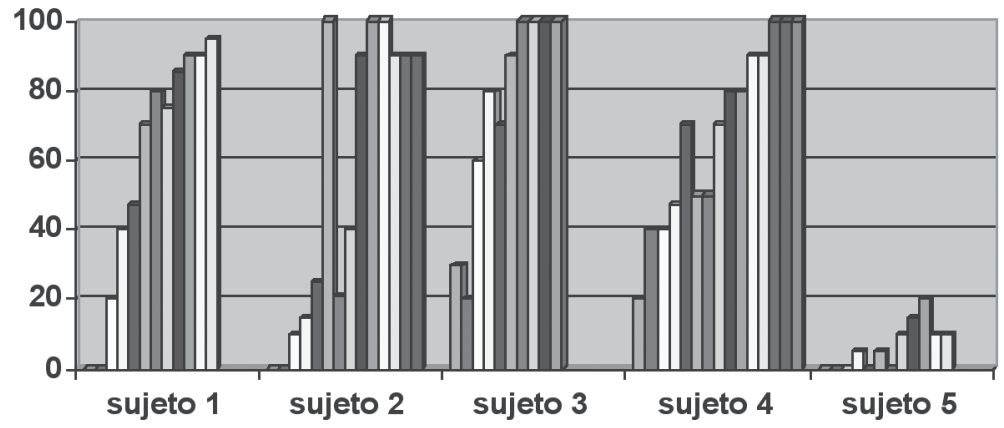

Tabla $\mathrm{N}^{\circ} 3$

Orden "Tóquese la boca"

Porcentajes de respuesta obtenidos por sesión

\begin{tabular}{|c|c|c|c|c|c|c|}
\hline & $\begin{array}{l}\mathrm{N}^{0} \text { de } \\
\text { Sesión }\end{array}$ & $\begin{array}{c}\text { Sujeto } \\
1\end{array}$ & $\begin{array}{c}\text { Sujeto } \\
2\end{array}$ & $\begin{array}{c}\text { Sujeto } \\
3\end{array}$ & $\begin{array}{c}\text { Sujeto } \\
4\end{array}$ & $\begin{array}{c}\text { Sujeto } \\
5\end{array}$ \\
\hline \multicolumn{7}{|l|}{ Línea } \\
\hline \multirow[t]{2}{*}{ base } & 1 & 20 & 10 & 50 & 60 & 0 \\
\hline & 2 & 40 & 10 & 60 & 40 & 0 \\
\hline \multirow{10}{*}{$\begin{array}{l}\text { inter- } \\
\text { vención }\end{array}$} & & & & & & \\
\hline & $\begin{array}{ll}n & 3 \\
& \end{array}$ & 45 & 15 & $\begin{array}{l}90 \\
70\end{array}$ & 80 & 0 \\
\hline & 5 & 70 & 40 & 80 & 40 & 5 \\
\hline & 6 & 75 & 50 & 70 & 90 & 5 \\
\hline & 7 & 90 & 80 & 100 & 100 & 10 \\
\hline & 8 & 80 & 90 & 100 & 100 & 10 \\
\hline & 9 & 95 & 90 & 100 & 100 & \\
\hline & 10 & 90 & 90 & 100 & 100 & \\
\hline & 11 & 90 & 100 & & & \\
\hline & 12 & & 100 & & & \\
\hline
\end{tabular}

No. de sesión

\begin{tabular}{|l|}
\hline$\square$ línea base 0 \\
$\square 2$ \\
$\square$ intervenc \\
$\square 4$ \\
$\square 5$ \\
$\square 6$ \\
$\square 7$ \\
$\square 8$ \\
$\square 9$ \\
$\square 10$ \\
\hline
\end{tabular}

Gráfico $\mathrm{N}^{\circ} 3$

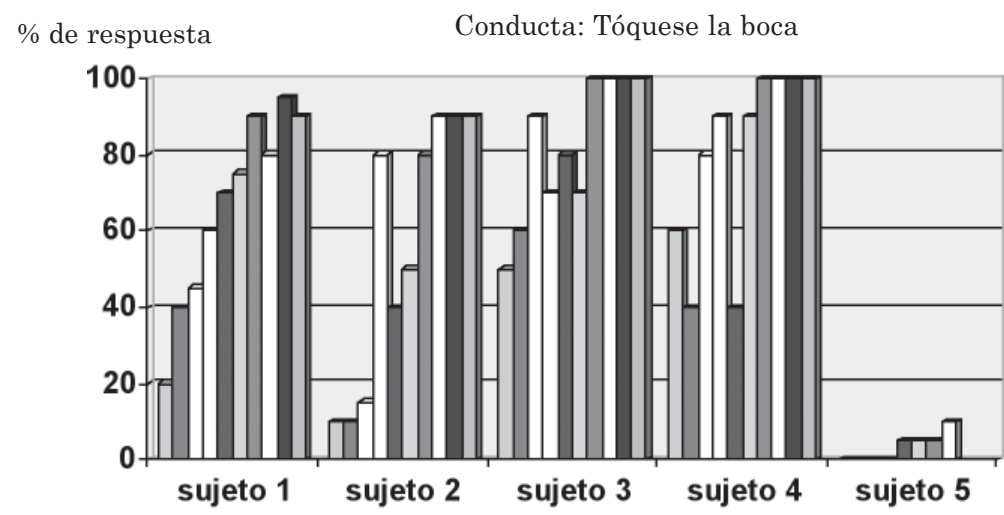

El concepto de boca se generalizó a otras personas y situaciones de la vida diaria. Llegó a formar parte del lenguaje expresivo de los niños. A la vez, permitió que pudieran seguir instrucciones, al dárseles la orden "véame la boca" "ábrala" "ciérrela”, preparándolo para la imitación de posiciones y emisión de sonidos, repertorio indispensable en la comunicación.

El avance y los tiempos de reacción para esta conducta, en los niños 1, 2 y 3 disminuyeron, no así para el niño $\mathrm{N}^{\circ} 4$, quien no había adquirido el concepto de yo, repertorio de entrada necesario para incorporar otros repertorios. La toma de conciencia y funcionalidad de las diferentes partes del
No. de sesión

\begin{tabular}{|l|}
\hline$\square 1$ \\
$\square 2$ \\
$\square 3$ \\
$\square 4$ \\
$\square 5$ \\
$\square 6$ \\
$\square 7$ \\
$\square 8$ \\
$\square 9$ \\
$\square 10$
\end{tabular}


Tabla $N^{\circ} 4$

Conducta: Tóquese los ojos

Porcentajes de respuesta por sesión

\begin{tabular}{lccccc}
\hline $\begin{array}{l}\text { Línea } \\
\text { base }\end{array}$ & $\begin{array}{c}\text { No de } \\
\text { Sesión }\end{array}$ & $\begin{array}{c}\text { Sujeto } \\
1\end{array}$ & $\begin{array}{c}\text { Sujeto } \\
2\end{array}$ & $\begin{array}{c}\text { Sujeto } \\
3\end{array}$ & $\begin{array}{c}\text { Sujeto } \\
4\end{array}$ \\
\hline \multirow{3}{*}{ Intervención } & 3 & 20 & 100 & 30 & 0 \\
& 1 & 0 & 50 & 0 & 0 \\
4 & 40 & 100 & 15 & 0 \\
5 & 30 & 100 & 10 & 2 \\
6 & 20 & 100 & 30 & 10 \\
7 & 60 & 100 & 40 & 5 \\
8 & 50 & & 50 & 20 \\
9 & 70 & & 60 & 20 \\
10 & 70 & & 80 & 10 \\
11 & 80 & & 90 & 20 \\
12 & 80 & & 90 & \\
13 & 80 & & 95 & \\
\hline
\end{tabular}

cuerpo son necesarias para montar sobre en ellas resto del aprendizaje.

Al finalizar la intervención los sujetos 1, 2, 3, 4 lograron identificar las diferentes partes del cuerpo. Para efectos de este trabajo, a manera de ilustración se grafican únicamente cuatro de ellas.

Como puede observarse, el número de sesiones para cada caso varía, así los sujetos 1, 2, 3 y 4 mostraron cambios en
Tabla $\mathrm{N}^{\circ} 5$

Tóquese cabeza

Porcentajes de respuesta por sesión

\begin{tabular}{lccccc}
\hline $\begin{array}{l}\text { No de } \\
\text { Sesión }\end{array}$ & Sujeto & Sujeto & Sujeto & Sujeto & Sujeto \\
\hline Línea & 1 & 2 & 3 & 4 & 5 \\
base & & & & & \\
1 & & & & & \\
2 & 0 & 0 & 20 & 20 & 0 \\
Intervenci. & 0 & 0 & 30 & 10 & 0 \\
3 & 70 & 20 & 70 & 50 & 0 \\
4 & 30 & 60 & 80 & 40 & 5 \\
5 & 52 & 60 & 90 & 60 & 10 \\
6 & 37 & 90 & 95 & 40 & 15 \\
7 & 54 & 92 & 80 & 90 & 0 \\
8 & 41 & 60 & 100 & 100 & 20 \\
9 & 64 & 60 & 100 & 100 & 10 \\
10 & 100 & 70 & 100 & 100 & 0 \\
11 & 90 & 90 & & & 0 \\
12 & 80 & 90 & & & 0 \\
& & & & & \\
\hline
\end{tabular}

las conductas tóquese la cabeza a partir de la sexta y sétima intervención. El sujeto número uno a partir de la novena, y el número 5 no adquirió ninguna de estas conductas.

Los programas de intervención conductual requieren de sistematización y constancia. Los resultados obtenidos por los

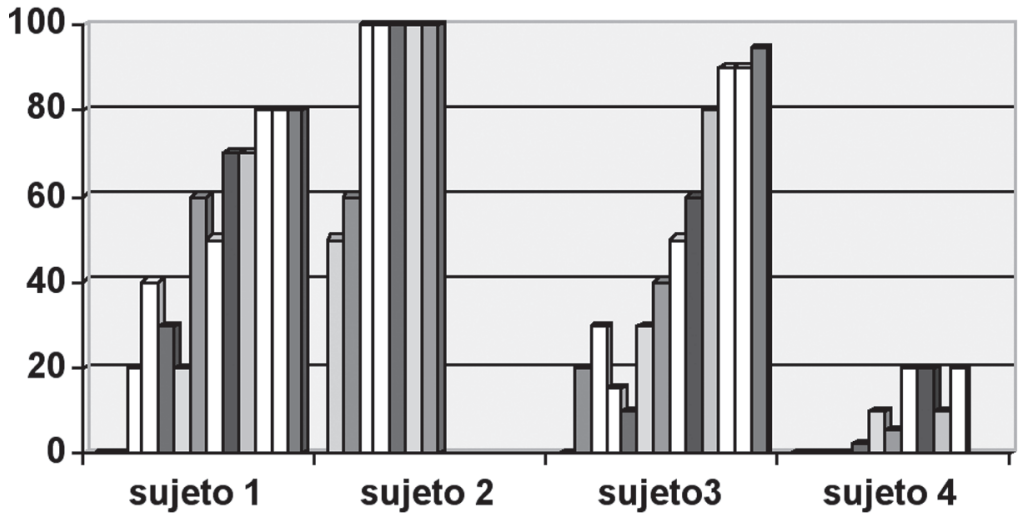




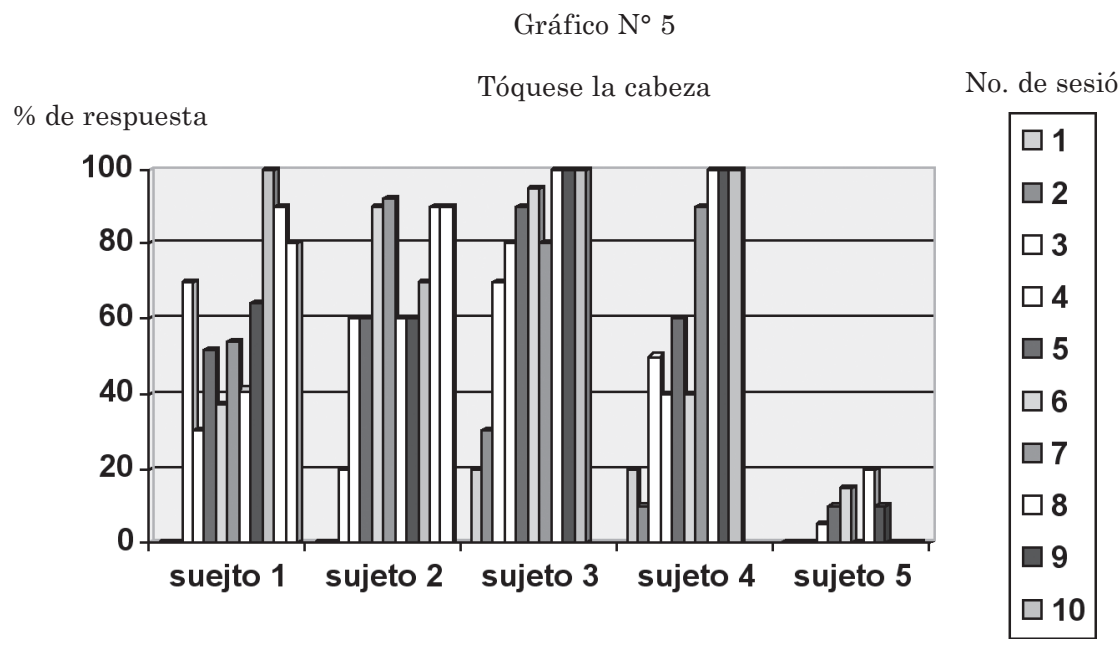

niños 1, 2, 3 y 4 se ven reforzados por una participación continua. Con el niño $\mathrm{N}^{\circ} 5$ se dieron a su vez dos condiciones adicionales. Una de ellas fue el grado de retardo mental asociado al autismo, sumado a esto, la falta de constancia en las sesiones, lo que entorpeció el proceso para la adquisición de cada una de las conductas trabajadas.

Los cambios mayores los encontramos con el concepto "tóquese la cabeza" probablemente porque la ejecución del movimiento para señalar la cabeza requiere de menor habilidad motora, por intervenir en el proceso los músculos gruesos del cuerpo, no así al tocar otras partes del cuerpo, como la nariz, la boca u ojos, cuya ejecución se asocia a movimientos finos, y la proximidad entre una parte del cuerpo y otra es menor, debiendo realizar el niño una acción más precisa.

Una vez que aprendieron a denominar las diferentes partes del cuerpo, se trabajó su funcionalidad, y con ello la expresión verbal.

De esta manera, el cuerpo se convirtió en el punto de partida para otros aprendizajes. El descubrimiento del cuerpo, la toma de conciencia del mismo y el apropiarse del espacio que les rodeaba a través de su control, les permitió establecer relaciones con las personas, los objetos y otras cosas que formaban parte de su entorno.
El lenguaje funcional se generó a partir de la identidad del yo como persona, de allí, la importancia del descubrimiento del cuerpo, su identidad y funcionalidad, además de la toma de conciencia y sus alcances.

La capacidad para expresarse y formular oraciones en los niños con autismo no es innata (Lovaas, 1985), al igual que el descubrimiento del cuerpo y la funcionalidad de cada de sus partes es una conducta que debe ser aprendida.

Estas conductas al igual que otras que han sido enseñadas se dividieron en sus componentes para una mejor efectividad en su adquisición.

\section{Conclusión}

El desarrollo del esquema corporal como punto de partida para la adquisición de los aprendizajes es fundamental en los niños con autismo, si a ello sumamos programas de intervención sistemáticos muy bien estructurados su avance es más efectivo. Al adquirir los niños el concepto de yo y la identificación de cada una de las partes del cuerpo, el concepto de funcionalidad de las mismas fue más fácil de incorporar, a su vez este descubrimiento facilitó la adquisición posteriormente de 
otras destrezas como: arriba, abajo, sobre, debajo, colores, formas y tamaños.

Los programas de reforzamiento, ayudan a incrementar conductas deseables en los niños con autismo, ya que si reforzamos adecuadamente una conducta, la probabilidad de que aumente su frecuencia es muy alta. Al determinarse que un reforzador funciona para una persona en una situación particular, ese mismo reforzador puede ser usado para incrementar otras habilidades que se deseen trabajar. Los resultados de este estudio muestran como los tiempos reacción iniciales se acortaron conforme se avanzaba, de esta manera el número de sesiones para adquirir resultados satisfactorios disminuyó entre una habilidad y otra.

Existe una estrecha relación entre el número de horas de entrenamiento que reciben los niños y la adquisición de repertorios conductuales en un tiempo menor

Un buen desarrollo del esquema corporal en los niños con autismo, les permitirá a su vez tomar conciencia de su propio cuerpo, establecer mejores relaciones con las personas que les rodean y, siendo una condición propia del autismo la dificultad para identificarse, un programa estructurado y sistemático tendiente a desarrollar estas habilidades se convertirá en básico para apoyar sobre él el resto del aprendizaje.

\section{Referencias bibliográficas}

Cabezas, H. "El niño con autismo: un programa estructurado para su educación. En Manual de Psicología Infantil”, España, Editorial Pirámide. Págs. 321-345. 2002.

Condemarín, M., Chadwick M., Milici N. "Madurez Escolar". Santiago de Chile, Editorial Andrés Bello. 1995.

Fernández, V. "Psicomotricidad como prevención e integración escolar" Revista de estudios y experiencias $\mathrm{N}^{\circ} 47,75$ 86 España. 1994.
Gallardo, J. Gallego J. Manual de logopedia Escolar, Ediciones Aljibe, Málaga España. 1993.

Hall, V. "El Manejo de la Conducta. El Modelo de Enseñanza Responsiva". Han H. Enterprises. New York. 1973.

Hernández. L. Aprendizaje y Problemas de Aprendizaje. Red Psicología, Artículos de Psicopedagogía. Galeón. hispavista.com. 2004.

Hobson, P. El autismo y el desarrollo de la mente. Editorial Alianza. Madrid España. 1995.

Kanner, L. Early infantile autism 1943-1955. American Journal of Orthopsychiatry, 26, 555-556. 1943.

Lovaas, I. "Experiemental studies in childhood schizophrenia. Building social behavior in autistic children by the use of electric shock". Journal of Experimental Reseach in Personality, (1965) 1, 99-109

Luria, A. Cerebro y lenguaje, Editorial Fontanella, S.A. Barcelona España. 1978.

Martos J. Riviere A. "Ministerio de Trabajo y Asuntos Sociales”, Madrid, España. 2001.

Merleau.M. Phenomenologie de la perception. N.R.F. Gallimard. En Vayer P. El niño frente al mundo. Editorial Científico Médica. México. 1977.

Polaino, A. Introducción al estudio científico del autismo infantil. Editorial Alambra, Madrid. 1982.

Ribes, E. Técnicas de Modificación de conducta y su aplicación al retardo deldesarrollo. Editorial Trillas, México. 1975. 
Riviére A. Martos J. El tratamiento del Autismo. Nuevas Perspectivas. Ministerio de Trabajo y Asuntos Sociales, Madrid, España. 1998.

Skinner, B. Science and Human Behavior. New York, Mcmillan Co. En:Blackham y Silberman A. Como Modificar la Conducta Infantil. Editorial Kapelusz. S.A. México. 1973.
Tinbergen, E. Early Childhood Autism: An etiological approach suplement. Journal of Comparative Ethology. U.S.A. 1972.

Vygotsky, El desarrollo de los procesos psicológicos superiores. Barcelona Crítica. En Gallardo R. Manual de Logopedia Escolar Ediciones Aljibe, Málaga España. 1993. 
